\title{
Smooth Interfaces for Spectral Element Approximations of Navier-Stokes Equations
}

\author{
Sha Meng ${ }^{1}$, Xin Kai Li ${ }^{2}$, and Gwynne Evans ${ }^{2}$ \\ 1 Department of Statistics, School of Mathematics, \\ University of Leeds, Leeds LS2 9JT, England \\ sha@amsta.leeds.ac.uk \\ 2 Institute of Simulation Sciences, Faculty of Computing Science and Engineering, \\ De Montfort University, Leicester LE1 9BH, England \\ xkl@dmu.ac.uk, gaevans@dmu.ac.uk
}

\begin{abstract}
A smoothing technique is developed to calculate the interface conditions of spectral element method for solving the incompressible Navier-Stokes equations. The first derivative of spectral element solution at the interface is calculated by using only the adjacent element information. Numerical simulations of an incompressible laminar fluid flow through a 2 : 1 planar contraction channel are presented for various Reynolds numbers.
\end{abstract}

Keywords: Spectral element method, A least square method, Navier-Stokes equations, Contraction channel flow

\section{Introduction}

Spectral element methods are high-order weighted residual techniques for the solution of partial differential equations typically in computational fluid dynamics [6]. Their success in the recent past in simulating complex flows derives from the flexibility of the method in representing accurately non-trivial geometries while preserving the good resolution properties of spectral method [1]. In the spectral element simulations, both the geometry and the solution are described through smooth functions so that the spectral element methods can obtain exponential accuracy by fully exploiting that regularity [6]. There are numerous fluid dynamics applications, however, where either very steep gradient of solutions or even discontinuous solutions are presented, e.g., a fluid through a channel with abrupt symmetrical contraction, interfaces in multiphase flows, or free surfaces in a die swell. A straightforward application of the spectral element methods in these situations may cause numerical instability as large errors induced by the discontinuous propagate in each element and eventually render the solution with oscillations everywhere. One reason for this instability phenomenon is that the spectral element method only enforces $C^{0}$ continuity at interfaces between each element. There have been methods proposed in which continuity of the first 
derivatives at the element interfaces is maintained [4], but they have the disadvantage that knowledge of a solution is required across the entire domain. When used on a parallel computer this translates into inter-processor communication which requires an extra amount of time.

In the current work we attempt to develop a spectral element scheme to approximate the interface conditions for the Gauss-Lobatto-Legendre polynomial approximations to the solutions of Navier-Stokes equations, and a smoothing technique to calculate the interface conditions at each element. The main idea presented here is to modify the Gauss-Lobatto-Legendre polynomial basis of the spectral element formulation by using a least square reconstruct procedure implemented on the first derivatives at interfaces of each element, in which the interface values can be calculated by using only the information on the adjacent elements. As a result the proposed interfacial smooth technique is implemented on the Navier-Stokes equations based on a channel flow with a symmetric abrupt contraction.

The paper is organized as follows: in Section 2 we introduce the basic idea upon which the spectral element formulation is based. In Section 3 we describe a smooth method to examine the accuracy between the numerical and analytical solution for the first derivative of trigonometric periodic function. Finally, the numerical simulation of the flow in a symmetric contraction channel is presented. The size of the salient corner vortex and the shape of stream function contours show a good agreement with the work of Dennis et al. [3] and Karageorghis et al. [5]. The separation length and the strength of the vortex increase as $R e$ increases after $R e>50$, and the downstream recirculations have been seen in the streamlines.

\section{Spectral element approximation}

The spectral element method is high-order weighted residual technique for the approximation of partial differential equations that combines the generality of finite element method with the accuracy of spectral method. In this section we will briefly describe the spectral element method based on a simple onedimensional Poisson equation defined by

$$
-u_{x x}=f, \quad x \in I=[a, b]
$$

with homogeneous Dirichlet boundary conditions

$$
u(a)=u(b)=0,
$$

where $f$ is a given function. Using the Galerkin technique, equations (1)-(2) can now be characterized by the following variational problem:

Find $u \in H_{0}^{1}(I)$, such that

$$
a(u, v)=(f, v), \quad \forall v \in H_{0}^{1}(I)
$$




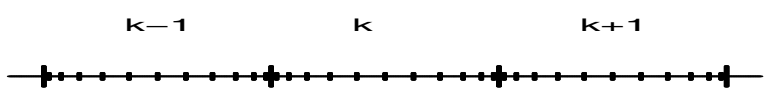

Fig. 1. Spectral element discretization in $1 \mathrm{D}$.

where

$$
\begin{gathered}
a(u, v)=\int_{a}^{b} u_{x}(x) v_{x}(x) d x, \quad(f, v)=\int_{a}^{b} f(x) v(x) d x, \\
H_{0}^{1}(I)=\left\{v \mid v \in L^{2}(I), v_{x} \in L^{2}(I), v(a)=v(b)=0\right\},
\end{gathered}
$$

$L^{2}(I)$ is the space of square integrable functions.

Following standard spectral element procedure, we begin, as usual, by introducing a family of partitions of $I$ such that $I=\cup_{k=1}^{K} I^{k}, \forall k, l, k \neq l, I_{k} \cap I_{l}=\emptyset$, where $K$ denotes the number of elements. In the development, we use $N$ as the degree of the polynomial. Fig. 1 shows the spectral element discretization on one dimensional geometry. On elements $k-1$ and $k$ the grid points are denoted $x_{0}^{k-1}, \ldots, x_{N}^{k-1}$ and $x_{0}^{k}, \ldots, x_{N}^{k}$, respectively. And then each element is mapped onto the parent element $\mathcal{I}=[-1,1]$ by using the equation

$$
x=\frac{L_{k}}{2} \xi^{k}+\frac{x_{L}^{k}+x_{R}^{k}}{2},
$$

where $x_{L}^{k}$ and $x_{R}^{k}$ denote the left and right coordinates of the elemental boundaries, $L_{k}$ is the element length, and $\xi \in \mathcal{I}$. The interpolant of $u(x)$ in the $k$ th element is then represented as

$$
u^{k}(\xi)=\sum_{i=0}^{N} u_{i}^{k} h_{i}\left(\xi^{k}\right)
$$

Here $u_{i}^{k}$ are nodal values of $u$, and $h_{i}\left(\xi^{k}\right)$ are the basis functions corresponding to element $k$ and node $i$, with property $h_{i}\left(\xi_{j}^{k}\right)=\delta_{i j}$. Expressions for these interpolants in terms of Chebyshev, Lengendre and other polynomials can be found in [2]. In this paper we choose the basis functions are the Gauss-LobattoLengendre polynomials defined as

$$
h_{i}(\xi)=-\frac{1}{N(N+1) L_{N}\left(\xi_{i}\right)} \frac{\left(1-\xi^{2}\right) L_{N}^{\prime}(\xi)}{\xi-\xi_{i}}, \quad \xi \in \mathcal{I},
$$

where $L_{N}(\xi)$ is the Legendre polynomial of degree $N, L_{N}^{\prime}(\xi)=d L_{N}(\xi) / d \xi$ and the $\xi_{i}$ are the Gauss-Lobatto-Legendre collocation points. Furthermore, there also exists a unique set of positive real numbers, $\rho_{i}$, corresponding with $\xi_{i}$, $(0 \leq i \leq N)$, such that the integration rule

$$
\int_{-1}^{1} \phi(\xi) d \xi=\sum_{i=0}^{N} \rho_{i} \phi\left(\xi_{i}\right)
$$

is exact for all polynomials $\phi(x)$ of degree $\leq(2 N-1)$ on the interval $[-1,1]$. We follow the standard spectral element method [6], an expansion of the function $u(x)$ can be written in terms of elements as 


$$
u(x)=\sum_{k=1}^{K} u^{k}(x)=\sum_{k=1}^{K} \sum_{i=0}^{N} u_{i}^{k} h_{i}(x)
$$

where $u_{i}^{k}$ are the point values for element $k$, and $x$ refers to the local coordinate. We require that the approximation function $u(x)$ is continuous through the interface of each element, i.e.,

$$
u^{k}\left(x_{N}\right)=u^{k+1}\left(x_{0}\right), \quad 1 \leq k \leq K-1
$$

and also satisfies the essential boundary condition

$$
u^{1}\left(x_{0}\right)=u^{K}\left(x_{N}\right)=0 .
$$

Expansion (8) together with the boundary conditions (9) and (10) are now inserted into the weak formulation (3) and the discrete equations are then generated by choosing appropriate functions $v$ which are unity at a point $\xi_{i}$ and zero at all other Gauss-Lobatto-Legendre points. In matrix form the spectral element procedure for equation (3) can be written as

$$
\mathbf{A u}=\mathbf{b}
$$

where $\mathbf{A}$ is the discrete Laplace operator and $\mathbf{b}$ is the right hand side vector with the boundary conditions.

It is clear that the approximation by spectral element discretization $u(x)$ defined by formulation (8) is only $C^{0}$ continuity and the first derivative may not continuous across each element. There has been a method proposed by Gottlieb et al. [4] in which continuity of the first derivatives at the sub-domain interfaces is maintained for the domain decomposition method. Following the same idea in [4], the element interfaces in the spectral element method can be calculated by enforcing $C^{1}$ continuity across each element interface, i.e., the first derivatives are calculated directly from formulation (8)

$$
\frac{d}{d x} u(x)=\sum_{k=1}^{K} \frac{d}{d x} u^{k}(x)=\sum_{k=1}^{K} \sum_{i=0}^{N} u_{i}^{k} \frac{d}{d x} h_{i}(x),
$$

and continuity of the first derivatives is enforced at each element interface

$$
\frac{d}{d x} u^{k}\left(x_{N}\right)=\frac{d}{d x} u^{k+1}\left(x_{0}\right), \quad \forall k \in\{1, \ldots, K-1\},
$$

Equation (13) implies that

$$
\sum_{i=0}^{N} u_{i}^{k} \frac{d}{d x} h_{i}\left(x_{N}\right)=\sum_{i=0}^{N} u_{i}^{k+1} \frac{d}{d x} h_{i}\left(x_{0}\right) .
$$


Although the continuity of the first derivative at each element interface is satisfied, the principal disadvantage of this enforcing method is that all of the elements are coupled together, which results in a relatively large amount of inter-processor communication and requires extra computer time to solve, in particular, in the parallel computational programs. The goal of this paper is to propose a simple modification for equation (14) so that the first derivative of the function $u(x)$ is almost continuous at each element interface but only using information from the adjacent elements in the approximation.

\section{Smoothing interface method}

In this section, we present a smoothing interface method (SIM) based on onedimensional spectral element approximations. Let $u^{k-1}(x)$ and $u^{k}(x)$ denote the approximations to the unknown variable $u(x)$ on elements $k-1$ and $k$, respectively, and they can be represented as

$$
u^{k-1}\left(x^{k-1}\right)=\sum_{i=0}^{N} u_{i}^{k-1} h_{i}\left(x^{k-1}\right), \quad u^{k}\left(x^{k}\right)=\sum_{i=0}^{N} u_{i}^{k} h_{i}\left(x^{k}\right),
$$

where $u_{i}^{k-1}$ and $u_{i}^{k}$ are the values of $u(x)$ in the grid points $x_{i}^{k-1}$ and $x_{i}^{k}$, respectively. Let $d^{k-1}(x)$ and $d^{k}(x)$ denote the first derivatives of $u^{k-1}(x)$ and $u^{k}(x)$, they can be written as

$$
d^{k-1}(x)=\sum_{i=0}^{N} d_{i}^{k-1} h_{i}\left(x^{k-1}\right), \quad d^{k}(x)=\sum_{i=0}^{N} d_{i}^{k} h_{i}\left(x^{k}\right),
$$

where $d_{i}^{k-1}, d_{i}^{k}$ are the values of the first derivatives of the variable $u(x)$ at the local nodes $x_{i}^{k-1}$ and $x_{i}^{k}$, respectively. The idea of the smoothing method is to find an alternative approach to calculate $d_{i}^{k-1}$ and $d_{i}^{k}$ by using the information from the adjacent elements $k-1$ and $k$ only. We wish to determine the best values for $d_{i}^{k}$ so that the deviations among $d_{i}^{k}$ and $\frac{d}{d x} u\left(x_{i}^{k}\right)$ are minimized. It turns out that to find a functional $\Phi$ such that

$$
\min \Phi\left(d_{0}^{k-1}, d_{1}^{k-1}, \ldots, d_{N}^{k-1}, d_{0}^{k}, \ldots, d_{N}^{k}, \lambda\right),
$$

where the functional $\Phi$ is given by

$$
\begin{gathered}
\Phi=\int_{k-1}\left(\left(d^{k-1}(x)-\frac{d}{d x} u^{k-1}(x)\right)^{2} d x+\int_{k}\left(d^{k}(x)-\frac{d}{d x} u^{k}(x)\right)^{2} d x\right. \\
+\lambda\left(d_{N}^{k-1}-d_{0}^{k}\right),
\end{gathered}
$$

and $\lambda$ is a parameter. If we substitute $d^{k}(x)$ as defined by equation (16) and $d u^{k}(x) / d x$ as defined by equation (14) into equation (18), we get 


$$
\begin{gathered}
\Phi=\int_{k-1}\left(\sum_{i=0}^{N} d_{i}^{k-1} h_{i}(x)-\sum_{i=0}^{N} u_{i}^{k-1} \frac{d}{d x} h_{i}(x)\right)^{2} d x \\
+\int_{k}\left(\sum_{i=0}^{N} d_{i}^{k} h_{i}(x)-\sum_{i=0}^{N} u_{i}^{k} \frac{d}{d x} h_{i}(x)\right)^{2} d x+\lambda\left(d_{N}^{k-1}-d_{0}^{k}\right) .
\end{gathered}
$$

We observe that $\Phi$ is an ordinary function of the unknowns $d_{i}^{k-1}$ and $d_{i}^{k}$ as reflected in our notation. To minimize $\Phi$, we need only to take its partial derivatives with respect to each unknown $d_{i}^{k-1}$ (as well as $d_{i}^{k}$ ) and set to zero. This implies that, at the minimum, all the partial derivatives $\partial \Phi / \partial d_{0}^{k-1}, \ldots, \partial \Phi / \partial d_{N}^{k-1}$ and $\partial \Phi / \partial d_{0}^{k}, \ldots, \partial \Phi / \partial d_{N}^{k}$ vanish. Writing the equations for these gives $2(N+2)$ equations:

$$
\begin{gathered}
\frac{\partial \Phi}{\partial d_{i}^{k-1}}=2 \int_{k-1}\left(\sum_{i=0}^{N} d_{i}^{k-1} h_{i}(x)-\sum_{i=0}^{N} u_{i}^{k-1} \frac{d}{d x} h_{i}(x)\right) h_{i}(x) d x=0, \quad i=0, \ldots, N-1, \\
\frac{\partial \Phi}{\partial d_{N}^{k-1}}=2 \int_{k-1}\left(\sum_{i=0}^{N} d_{i}^{k-1} h_{i}(x)-\sum_{i=0}^{N} u_{i}^{k-1} \frac{d}{d x} h_{i}(x)\right) h_{N}(x) d x+\lambda=0, \\
\frac{\partial \Phi}{\partial d_{i}^{k}}=2 \int_{k}\left(\sum_{i=0}^{N} d_{i}^{k} h_{i}(x)-\sum_{i=0}^{N} u_{i}^{k} \frac{d}{d x} h_{i}(x)\right) h_{i}(x) d x=0, \quad i=1, \ldots, N, \\
\frac{\partial \Phi}{\partial d_{0}{ }^{k}}=2 \int_{k}\left(\sum_{i=0}^{N} d_{i}^{k} h_{i}(x)-\sum_{i=0}^{N} u_{i}^{k} \frac{d}{d x} h_{i}(x)\right) h_{0}(x) d x-\lambda=0 .
\end{gathered}
$$

Solving these equations by using the integration formulation (7), we obtain

$$
\begin{gathered}
d_{i}^{k-1}=\frac{1}{J^{k-1}} \sum_{j=0}^{N} u_{j}^{k-1} \frac{d}{d \xi} h_{j}\left(\xi_{i}\right), \quad i=0, \ldots, N-1, \\
d_{i}^{k}=\frac{1}{J^{k}} \sum_{j=0}^{N} u_{j}^{k} \frac{d}{d \xi} h_{j}\left(\xi_{i}\right), \quad i=1, \ldots, N \\
d_{N}^{k-1}=d_{0}^{k}=\frac{1}{J^{k-1}+J^{k}} \sum_{j=0}^{N}\left(u_{j}^{k-1} \frac{d}{d \xi} h_{j}\left(\xi_{N}\right)+u_{j}^{k} \frac{d}{d \xi} h_{j}\left(\xi_{0}\right)\right),
\end{gathered}
$$

where $J^{k-1}$ and $J^{k}$ are the values of Jacobian that come from mapping subdomains $\Omega_{k-1}$ and $\Omega_{k}$ onto the parent element $\chi^{2}=[-1,1]$. With the above equations (20)-(22), the interface of elements can be calculated in the sense of 
least-square approximations by using only information from the adjacent elements $k-1$ and $k$. It is important to realize that the deviations squared of the first derivatives should continually decrease as the degree of the polynomial is raised. If the approach is implemented on a parallel computer the only communication that is required is between adjacent processors.

Before leaving this section, we show some numerical experiments which illustrate the accuracy of the spectral element method with smoothing interface strategy. We computed the first derivatives of the test function

$$
f(x)=|\sin (x)|, \quad-\pi \leq x \leq \pi .
$$

Its first derivative is

$$
\frac{d}{d x} f(x)= \begin{cases}-\cos (x), & -\pi \leq x \leq 0 \\ \cos (x), & 0 \leq x \leq \pi\end{cases}
$$

Clearly, the first derivative function $d f(x) / d x$ is discontinuous at $x=0$. How well does the smoothing interface method do on such a function? Fig. 2 shows the exact and numerical solutions for the first derivatives $d f(x) / d x$ with different numbers of Gauss-Lobatto-Legendre collocation points as well as different numbers of the elements. As illustrated in Fig. 2, the numerical solutions agreed well with the exact solutions when the smoothing interface technique is used. It is also important to note that the smoothing solutions do not have any oscillations at $x=0$ where the first derivative function $d f(x) / d x$ is discontinuous. Table 1 shows that the numerical solutions calculated with and without smooth interface technique by using four elements and twentyfour collocation points on each element. The data show that both algorithms performed well for the discontinuous function $d f(x) / d x$. It is quite self-evident that the smoothing solutions (SIM) are more accurate than the solutions without smoothing technique (SEM). This can be seen in Table 1 when $x=-\pi / 2$ and $x=\pi / 2$ the error for the SIM solutions is $10^{-13}$, but it is only $10^{-6}$ for the SEM solutions.

Table 1. A comparison among the analytical, SIM and SEM solutions with 24 collocation points and 8 elements.

\begin{tabular}{|c|c|c|c|}
\hline$x$ & Analytical solution of $f^{\prime}(x)$ & SIM solution of $f^{\prime}(x)$ & SEM solution of $f^{\prime}(x)$ \\
\hline$-\frac{3}{4} \pi$ & $.70710670 E+00$ & $.70710670 E+00$ & $.70710680 E+00$ \\
\hline$-\frac{1}{2} \pi$ & $-.75497900 E-07$ & $-.75497890 E-07$ & $.36091110 E-07$ \\
\hline$-\frac{1}{4} \pi$ & $-.70710680 E+00$ & $-.70710680 E+00$ & $-.70710670 E+00$ \\
\hline$\frac{1}{4} \pi$ & $.70710680 E+00$ & $.70710680 E+00$ & $.70710690 E+00$ \\
\hline$\frac{1}{2} \pi$ & $.75497900 E-07$ & $.75497890 E-07$ & $.18708690 E-06$ \\
\hline$\frac{3}{4} \pi$ & $-.70710670 E+00$ & $-.70710670 E+00$ & $-.70710660 E+00$ \\
\hline
\end{tabular}

\section{Numerical results}

In this section, numerical results are presented for the incompressible NavierStokes flows in a $2: 1$ contraction channel by using the spectral element method 


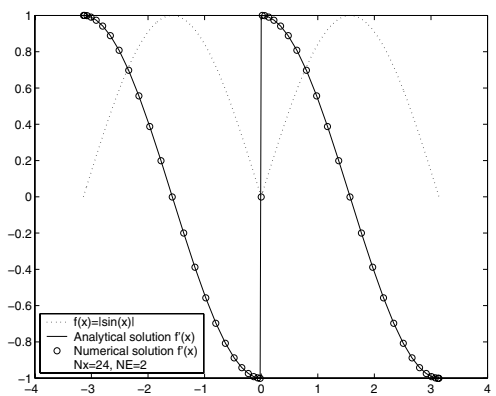

(a)

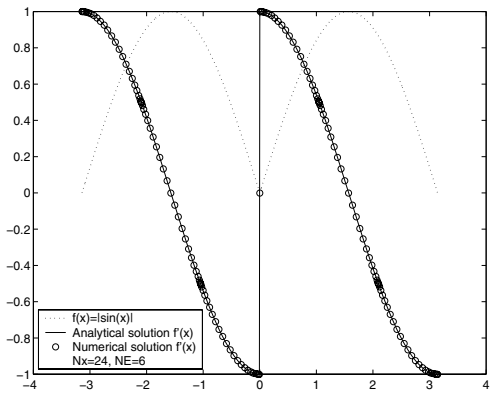

(c)

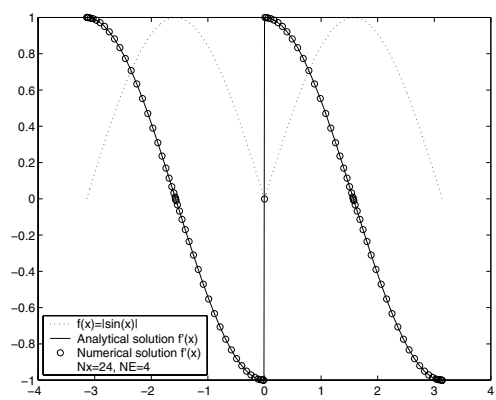

(b)

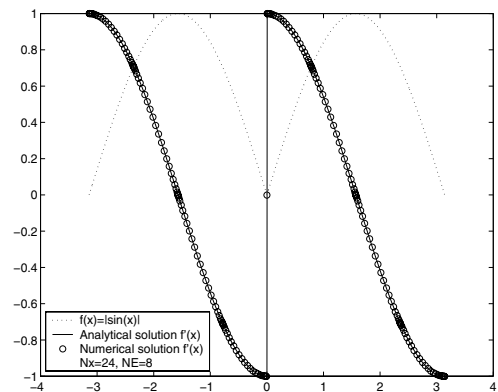

(d)

Fig. 2. Numerical solutions with the smoothing interface technique compare with the analytical solutions by using different numbers of collocation points $(\mathrm{Nx})$ and elements $(\mathrm{NE})$.

with smoothing interface technique. The incompressible Navier-Stokes equations are given by:

$$
\begin{gathered}
\frac{\partial \mathbf{u}}{\partial t}+\mathbf{u} \cdot \nabla \mathbf{u}-\frac{1}{R e} \triangle \mathbf{u}+\nabla p=\mathbf{f}, \\
-\nabla \cdot \mathbf{u}=0,
\end{gathered}
$$

where $\mathbf{u}=(u, v)$ is the velocity, $\mathrm{p}$ is the pressure, $\mathbf{f}$ is a given function, and $R e=\rho U L / \eta$ is Reynolds number defined the properties of the fluid.

\subsection{Planar contraction flow}

The $2: 1$ contraction channel flow geometry is shown in Fig. 3. Here the fluid enters upstream in the channel as a fully developed parabolic profile, and exits far downstream as a flat liquid sheet. The $2: 1$ planar contraction channel is chosen in order to compare with the results already published in Dennis et al. [3] and Karageorghis et al. [5]. In such a geometrical channel, the height of the inflow half channel is taken to be unit and the height of the outflow channel is $a=1 / 2$, and the total length of the channel is 4 .

Two non-uniform different meshes shown in Fig. 4 were used in the numerical simulations. Mesh 1 has three elements, twelve collocation points in the $x$ direction and six collocation points in the $y$ direction in each element, while there are 


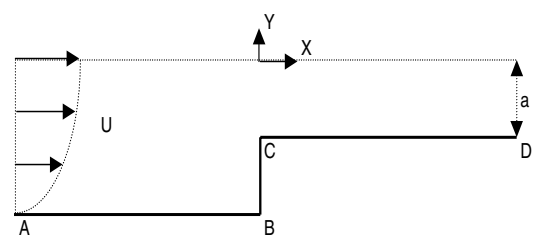

Fig. 3. The $2: 1$ planar contraction flow geometry.

three elements, sixteen and eight collocation points in the $x$ and $y$ directions on each element, respectively, in Mesh 2.

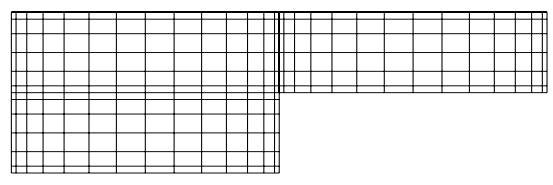

$\mathrm{Nx}=14, \mathrm{Ny}=6, \mathrm{NE}=3$

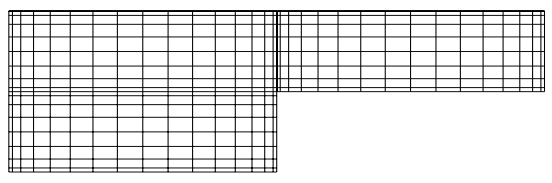

$\mathrm{Nx}=16, \mathrm{Ny}=8, \mathrm{NE}=3$

Fig. 4. Meshes for the 2: 1 planar contraction channel problem.

Table 2 shows the length $l_{1}$ and the width $l_{2}$ of the salient corner vortex with different Reynolds numbers on Mesh 1 and Mesh 2. We can see that the size of corner vortex diminishes as $R e$ increases from $R e=0$ to $R e=50$, and then begins to grow slowly with $R e>50$. In Table 3 , we compare the values of $l_{1}$ and $l_{2}$ for various Reynolds numbers on Mesh 2 with the results obtained by Dennis et al. [3] and Karageorghis et al. [5]. Table 3 shows that our numerical results are in good agreement with those results. Also, we find that $l_{1}$ grows more quickly than $l_{2}$ when $R e$ increases from 50 to 200, which implies that the corner vortex grows in size along the upstream channel more quickly than up the wall at $x=0$ as $R e$ increases.

\begin{tabular}{|l|r|r|r|r|r|r|r|r|}
\hline & & $R e=0$ & $R e=1$ & $R e=10$ & $R e=50$ & $R e=100$ & $R e=150$ & $R e=200$ \\
\hline$l_{1}$ & Mesh 1 & 0.2548 & 0.2201 & 0.1477 & 0.1280 & 0.1379 & 0.1576 & 0.1870 \\
\hline & Mesh 2 & 0.2610 & 0.2399 & 0.1545 & 0.1077 & 0.1389 & 0.1467 & 0.1825 \\
\hline$l_{2}$ & Mesh 1 & 0.3086 & 0.2793 & 0.1767 & 0.1215 & 0.1328 & 0.1474 & 0.1621 \\
\hline & Mesh 2 & 0.3181 & 0.2954 & 0.1706 & 0.1396 & 0.1298 & 0.1494 & 0.1706 \\
\hline
\end{tabular}

Table 2. Values of $l_{1}$ and $l_{2}$ for various $R e$ numbers on the Mesh 1 and Mesh 2 .

Contours of the stream function for $R e=0,10,50,100,150,200$ on Mesh 2 are plotted in Fig. 5. The streamline plots give a qualitatively satisfactory to the flow solutions, we can see that the eddy of recirculation is happened for the Stokes flow at $R e=0$, and as $R e$ increases from zero, the length of corner vortex $l_{1}$ initially decreases until at $R e=50$. For higher value $R e$ number, the eddy length $l_{1}$ increases monotonically with $R e$. This phenomenon shows that the vortex develops as $R e$ is increased. 


\begin{tabular}{|c|c|c|c|c|c|c|c|c|}
\hline & & $R e=0$ & $R e=1$ & $R e=10$ & $R e=50$ & $R e=100$ & $R e=150$ & $R e=200$ \\
\hline$l_{1}$ & (a) & 0.2610 & 0.2399 & 0.1545 & 0.1077 & 0.1389 & 0.1467 & 0.1825 \\
\hline & (b) & 0.268 & 0.243 & 0.153 & 0.128 & 0.138 & - & - \\
\hline & (c) & 0.285 & - & 0.150 & 0.129 & 0.143 & 0.160 & 0.183 \\
\hline$l_{2}$ & (a) & 0.3181 & 0.2954 & 0.1706 & 0.1396 & 0.1298 & - & - \\
\hline & (b) & 0.311 & 0.281 & 0.163 & 0.124 & 0.122 & - & - \\
\hline
\end{tabular}

Table 3. The length and the width of the salient corner vortex for (a) smoothing interface spectral element method; (b) finite-difference scheme of Dennis et al. [3]; (c) spectral collocation method of Karageorghis et al. [5].
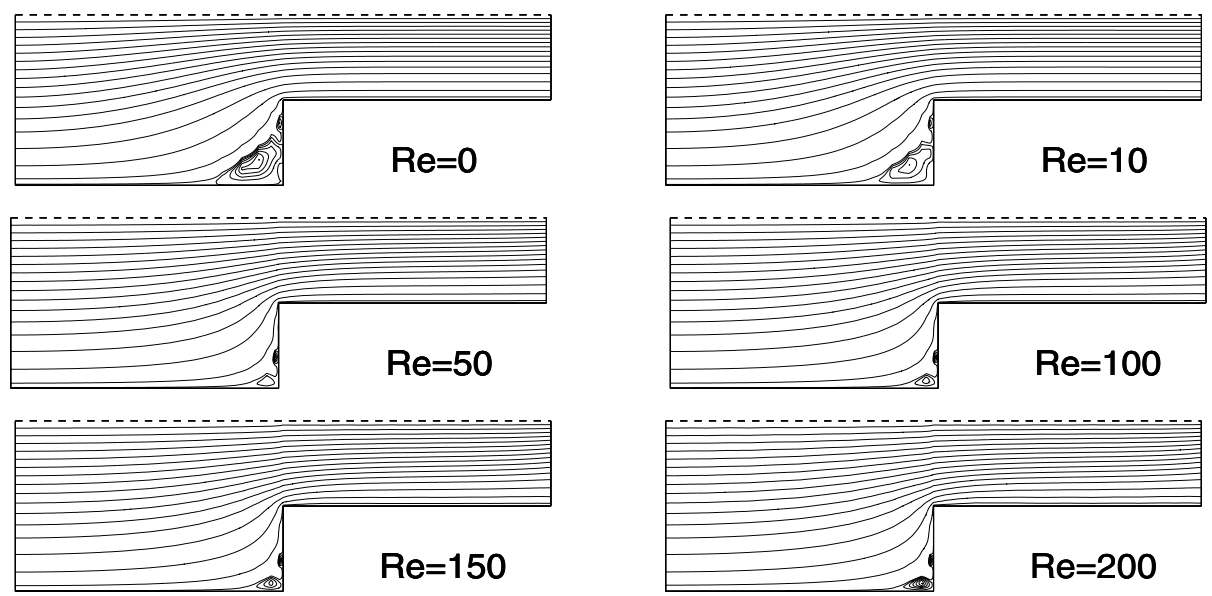

Fig. 5. Flow streamlines for various $R e$ numbers on Mesh 2.

\section{Acknowledgements}

Sha Meng gratefully acknowledges the financial support of Ph.D scholarship of De Montfort University of England.

\section{References}

1. C. Canuto, M. Hussaini, A. Quarteroni, and T. Zang. Spectral methods in Fluid Dynamics. Springer-Verlag, New York, 1987.

2. S. A. Orszag D. Gottlieb. Numerical Analysis of Spectral methods: Theory and Applications. SIAM, Philadelphia, 1977.

3. S. C. R. Dennis and F. T. Smith. Stesdy flow through a channel with a symmetrical constriction in the form of a step. Proc. Roy. Soc. London A, 372:393-414, 1980.

4. D. Gottlieb and R. S. Hirsh. Parallel pseudospectral domain decomposition techniques. J. Sci. Comput, 4:309-325, 1989.

5. A. Karageorghis and T. N. Phillips. Conforming Chebyshev spectral methods for the solution of Laminar flow in a constricted channel. IMA J. Numer. Anal, 11:33-54, 1991.

6. Y. Maday and A. T. Patera. Spectral element methods for the incompressible Navier-Stokes equations. in State of the Art Surverys in Computational Mechanics, pages 71-143, 1989. 